\title{
Quark condensate in a weak magnetic field
}

\author{
Christoph P. Hofmann \\ Facultad de Ciencias, Universidad de Colima, Bernal Díaz del Castillo 340, Colima C.P. 28045, Mexico
}

(Received 25 October 2017; published 18 January 2019)

\begin{abstract}
The low-temperature representation for the quark condensate in a weak magnetic field $H$ is known up to two-loop order. Remarkably, at one-loop order, the published series for the quark condensate in the chiral limit and $H \ll T^{2}$ are inconsistent. Using an alternative representation for the kinematical Bose functions, we derive the series to arbitrary order in $H / T^{2}$ and show that both published results are incorrect.
\end{abstract}

DOI: $10.1103 /$ PhysRevD.99.014030

\section{INTRODUCTION}

The low-energy behavior of quantum chromodynamics in the presence of a magnetic field has been explored by many authors in great detail. In particular, the quark condensate at finite temperature and magnetic field has been calculated with different techniques. These include lattice QCD [1-7], chiral perturbation theory [8-13], the Nambu-Jona-Lasinio model and its extensions [14-17], and yet other methods [18-21]. Likewise, the related phenomena of magnetic catalysis and inverse magnetic catalysis have been studied in Refs. [22-31]. A nice review of QCD in a magnetic field is provided by Andersen et al. [32].

Of particular interest in our study is the low-temperature expansion of the quark condensate in the chiral limit in a weak magnetic field $H$. Remarkably, the corresponding series provided by the author of Refs. [8-11] and the author of Refs. [12,13] are inconsistent. The motivation for the present study is to decide which of the two published results is correct, and to go to higher orders in the weak magnetic field expansion. Our calculation is based on chiral perturbation theory, much like Refs. [8-13], but it relies on an alternative representation for the kinematical Bose functions that appear at one-loop order.

As it turns out, the series we derive below is different from both of the two published results. We have checked that our series perfectly coincides with the exact result that we have evaluated numerically. We stress that criticism is not directed towards the one-loop evaluation of the partition function, where the many different calculations all lead to the same result. Rather, our intention is to point out that, in the low-temperature expansion of the quark condensate at

Published by the American Physical Society under the terms of the Creative Commons Attribution 4.0 International license. Further distribution of this work must maintain attribution to the author(s) and the published article's title, journal citation, and DOI. Funded by SCOAP ${ }^{3}$. one-loop order, errors exist concerning the weak magnetic field expansion $H \ll T^{2}$ in the chiral limit. More importantly, the correct series is derived in the present study for the first time.

It should be emphasized that the errors in the published series cannot be "detected" in the many articles [1-31] that also deal with the finite-temperature quark condensate in a magnetic field. The point is that the chiral limit in these references is implemented by numerically extrapolating the exact one-loop result (that is valid for arbitrary finite pion masses $M$ ) to the case $M \rightarrow 0$ - and not by using a series expansion for the chiral limit. Note also that, away from the chiral limit, there are no discrepancies or errors: all different calculations concerning the one-loop result coincide. Still, in view of the fact that the quark condensate is a fundamental quantity in quantum chromodynamics, we believe it is important to provide the (correct) lowtemperature series for this quantity in a weak magnetic field in the chiral limit.

The article is organized as follows: In Sec. II, we review the two published results that refer to the low-temperature expansion of the quark condensate in weak magnetic fields in the chiral limit. In Sec. III, the one-loop evaluation of the quark condensate within chiral perturbation theory-based on an alternative representation for the Bose functions-is presented, and the weak magnetic field expansion in the chiral limit is derived. We then compare our series with the two published results in Sec. IV, and finally conclude in Sec. V. Technical aspects of the evaluation are relegated to two Appendixes. In Appendix A, chiral perturbation theory calculations are discussed explicitly. In Appendix B, we provide the (analytical) connection between the representation for the Bose-Einstein functions used in the literature (Refs. [8-13]) and the one used here.

\section{PUBLISHED SERIES FOR THE CHIRAL LIMIT}

Our analysis concerns the low-temperature expansion of the quark condensate in the chiral limit in a weak magnetic 
field. The weak magnetic field limit is implemented by $|q H| \ll T^{2}$, where $|q|$ stands for the electric charge of the pion. The relevant quantity is

$$
\frac{\langle\bar{q} q\rangle}{\langle 0|\bar{q} q| 0\rangle},
$$

where $\langle 0|\bar{q} q| 0\rangle$ is the quark condensate at zero temperature and zero magnetic field. Two series are available in the literature for the above quantity in the chiral limit: up to one-loop order, the author of Refs. [8-11] obtains

$$
\begin{aligned}
\frac{\langle\bar{q} q\rangle}{\langle 0|\bar{q} q| 0\rangle}= & 1+\frac{\mathcal{C}|q H|}{16 \pi^{2} F^{2}}-\frac{T^{2}}{8 F^{2}}-\frac{7 \sqrt{|q H|} T}{48 \pi F^{2}} \\
& -\frac{|q H|}{16 \pi^{2} F^{2}} \log \frac{|q H|}{T^{2}}, \quad|q H| \ll T^{2}, \\
\mathcal{C}= & \log 2-2 \gamma_{E}+2 \log 4 \pi+\frac{1}{3},
\end{aligned}
$$

while the author of Refs. [12,13] ends up with

$$
\begin{aligned}
\frac{\langle\bar{q} q\rangle}{\langle 0|\bar{q} q| 0\rangle} & =1+\frac{|q H| \log 2}{16 \pi^{2} F^{2}}-\frac{T^{2}}{8 F^{2}}+\frac{5 \sqrt{|q H|} T}{48 \pi F^{2}}+\cdots, \\
|q H| & \ll T^{2} .
\end{aligned}
$$

Both series contain the leading term at zero temperature,

$$
\frac{|q H| \log 2}{16 \pi^{2} F^{2}},
$$

which is linear in the magnetic field and positive, and has been derived in the pioneering paper by Shushpanov and Smilga [33]. ${ }^{1}$ As far as finite-temperature corrections are concerned, we first have a term that does not involve the magnetic field,

$$
-\frac{T^{2}}{8 F^{2}}
$$

derived a long time ago in the original article by Gasser and Leutwyler [34]. However, in a nonzero magnetic field, the two series disagree with respect to the leading contribution at finite temperature: the coefficients of the $\sqrt{H} T$ term are different both in magnitude and in sign. Finally, according to Refs. [8-11], logarithmic terms of the form $H \log \left(H / T^{2}\right)$ also emerge.

In order to make the low-temperature expansion in the weak magnetic field limit $\left(|q H| \ll T^{2}\right)$ more transparent, we factorize out temperature and use the relevant expansion parameter $\epsilon<1$,

$$
\epsilon=\frac{|q H|}{T^{2}}
$$

\footnotetext{
${ }^{1}$ Note that the constant $\mathcal{C}$ in Eq. (2.2) involves further terms: $-2 \gamma_{E}+2 \log 4 \pi+\frac{1}{3}$. As we comment at the end of Sec. IV, these terms should not appear at zero temperature.
}

The two published series can then be cast into the general form

$$
\begin{aligned}
\frac{\langle\bar{q} q\rangle}{\langle 0|\bar{q} q| 0\rangle}= & 1+\frac{|q H| \log 2}{16 \pi^{2} F^{2}}+\left\{q_{1} \sqrt{\epsilon}+q_{2} \epsilon \log \epsilon+q_{3} \epsilon\right. \\
& \left.+q_{4} \epsilon^{2}+q_{5} \epsilon^{3}+\mathcal{O}\left(\epsilon^{4}\right)\right\} T^{2}-\frac{1}{8 F^{2}} T^{2}+\mathcal{O}\left(T^{4}\right)
\end{aligned}
$$

Let us consider the quantity

$$
\begin{aligned}
Q(\epsilon)= & \frac{1}{\sqrt{\epsilon} T^{2}}\left(\frac{\langle\bar{q} q\rangle}{\langle 0|\bar{q} q| 0\rangle}-1-\frac{|q H| \log 2}{16 \pi^{2} F^{2}}\right. \\
& \left.+\frac{1}{8 F^{2}} T^{2}-\mathcal{O}\left(T^{4}\right)\right) .
\end{aligned}
$$

In the limit $\epsilon \rightarrow 0$, we have

$$
\lim _{\epsilon \rightarrow 0} Q(\epsilon)=q_{1} .
$$

Irrespective of whether or not a logarithmic contribution is present, $Q(\epsilon)$ should converge to the leading coefficient $q_{1}$. The authors of Refs. [8-11] and Refs. [12,13] end up with different values for $q_{1}$. This is our starting point. As it turns out, our leading coefficient $q_{1}$ is yet different from both of the published results, and higher-order terms in our series disagree with the Agasian series [8-11]. Our approach relies on chiral perturbation theory, and the specific representation for the kinematical functions we chose allows for a systematic and transparent expansion in the limit $|q H| \ll T^{2}$.

\section{CHIRAL PERTURBATION THEORY EVALUATION}

The essentials of chiral perturbation theory have been outlined in many excellent reviews, to which the interested reader is referred (see, e.g., Refs. [35,36]). Here we merely provide a brief sketch of the method and discuss the oneloop evaluation.

The QCD Lagrangian for two flavors reads

$$
\mathcal{L}_{\mathrm{QCD}}=-\frac{1}{2 g^{2}} \operatorname{tr}_{c} G_{\mu \nu} G^{\mu \nu}+\bar{q} i \gamma^{\mu} D_{\mu} q-\bar{q} m q, \quad(q=u, d) .
$$

In the present study, we focus on the isospin limit $m_{u}=m_{d}$. The quark condensate,

$$
\langle 0|\bar{q} q| 0\rangle,
$$

is the order parameter associated with the spontaneously broken chiral symmetry $S U(2) \times S U(2) \rightarrow S U(2)$. The corresponding Goldstone bosons are the three pions. 
In the effective field theory, the pion fields $\pi^{i}(i=1,2,3)$ are contained in the $S U(2)$ matrix $U=\exp \left(i \tau^{i} \pi^{i} / F\right)$, where $\tau^{i}$ are the Pauli matrices and $F$ is the (tree-level) pion decay constant. The leading term in the effective Lagrangian is of momentum order $p^{2}$ and reads

$$
\mathcal{L}_{\text {eff }}^{2}=\frac{1}{4} F^{2} \operatorname{Tr}\left[\left(D_{\mu} U\right)^{\dagger}\left(D_{\mu} U\right)-M^{2}\left(U+U^{\dagger}\right)\right],
$$

where $M$ is the (tree-level) pion mass. It should be pointed out that the magnetic field $H$ is taken into account by the covariant derivative

$$
D_{\mu} U=\partial_{\mu} U+i[Q, U] A_{\mu}^{\mathrm{EM}}
$$

$Q$ stands for the charge matrix of the quarks, $Q=$ $\operatorname{diag}(2 / 3,-1 / 3) e$, and the gauge field $A_{\mu}^{\mathrm{EM}}=(0,0$, $-H x, 0)$ incorporates the constant magnetic field in Landau gauge [32]. The next-to-leading piece in the effective Lagrangian- $\mathcal{L}_{\text {eff }}^{4}$-is of momentum order $p^{4}$, and it involves various next-to-leading-order effective constants $l_{i}$ and $h_{i}$ that require renormalization (see Appendix A). The explicit form of $\mathcal{L}_{\text {eff }}^{4}$ can be found, e.g., in Refs. [36,37].

Chiral perturbation theory refers to low temperatures, small quark masses and weak magnetic fields. We first consider the free energy density, from which the quark condensate can be derived. The corresponding Feynman diagrams, up to one-loop order $p^{4}$, are depicted in Fig. 1. Their evaluation leads to the following low-temperature representation for the free energy density ${ }^{2}$ :

$z=z_{0}(M, 0, H)-\frac{3}{2} g_{0}(M, T, 0)-\tilde{g}_{0}(M, T, H)+\mathcal{O}\left(p^{6}\right)$.

The quantity $z_{0}$ is the free energy density at zero temperature, while the two other contributions are finite-temperature corrections: the first one refers to zero magnetic field, and the second one incorporates the magnetic field.

As we show in Appendix A, the quark condensate can then be obtained from the free energy density. Up to one-loop order, we get

$$
\begin{aligned}
\frac{\langle\bar{q} q\rangle}{\langle 0|\bar{q} q| 0\rangle}= & 1-\frac{|q H|}{16 \pi^{2} F^{2}} \int_{0}^{\infty} d t t^{-1}\left(\frac{1}{\sinh (t)}-\frac{1}{t}\right) \\
& -\frac{3 g_{1}(0, T, 0)}{2 F^{2}}-\frac{\tilde{g}_{1}(0, T, H)}{F^{2}}+\mathcal{O}\left(p^{4}\right),
\end{aligned}
$$

\footnotetext{
${ }^{2}$ The present evaluation parallels the evaluation of the partition function in zero magnetic field, described in much detail in Refs. [38,39].
}
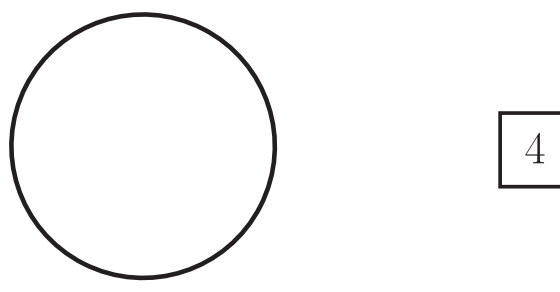

$4 A$

$4 B$

FIG. 1. QCD partition function diagrams contributing up to one-loop order in the low-temperature expansion. The filled circle refers to $\mathcal{L}_{\text {eff }}^{2}$, while the number 4 in the box corresponds to $\mathcal{L}_{\text {eff }}^{4}$.

where $\langle 0|\bar{q} q| 0\rangle$ is the quark condensate at zero temperature and zero magnetic field. ${ }^{3}$ The first line in Eq. (3.6) refers to zero temperature, while the kinematical functions $g_{1}(0, T, 0)$ and $\tilde{g}_{1}(0, T, H)$ describe the behavior of the system at finite temperature. We now analyze in detail the structure of the above terms.

The basic object in the evaluation of the partition function-or, equivalently, the free energy density-is the thermal propagator $G(x)$ for the pions in the background of a magnetic field. It can be constructed from the zero-temperature propagator $\Delta(x)$ in Euclidean space by

$$
G(x)=\sum_{n=-\infty}^{\infty} \Delta\left(\vec{x}, x_{4}+n \beta\right), \quad \beta=\frac{1}{T} .
$$

The propagator $\Delta^{0}(x)$, referring to the neutral pion, is not affected by the magnetic field and takes the simple form

$$
\begin{aligned}
\Delta^{0}(x) & =(2 \pi)^{-d} \int \mathrm{d}^{d} p e^{i p x}\left(M^{2}+p^{2}\right)^{-1} \\
& =\int_{0}^{\infty} \mathrm{d} \rho(4 \pi \rho)^{-d / 2} e^{-\rho M^{2}-x^{2} / 4 \rho} .
\end{aligned}
$$

As for the two charged pions, it is convenient to start with the representation for the zero-temperature propagator in Minkowski space given in Refs. [32,40]:

$$
\begin{aligned}
\Delta^{ \pm}(x)= & \exp \left[i s_{\perp} \Phi\left(x_{\perp}\right)\right] \int \frac{d^{4} p}{(2 \pi)^{4}} e^{-i p x} \Delta^{ \pm}\left(p_{\|}, p_{\perp}\right), \\
\Delta^{ \pm}\left(p_{\|}, p_{\perp}\right)= & i \int_{0}^{\infty} \frac{d s}{\cos (|q H| s)} \exp \left(i s\left(p_{\|}^{2}-M^{2}\right)\right. \\
& \left.-i p_{\perp}^{2} \frac{\tan (|q H| s)}{|q H|}\right)
\end{aligned}
$$

where

\footnotetext{
${ }^{3}$ At one-loop order, up- and down-quark condensates coincide: $\langle\bar{u} u\rangle=\langle\bar{d} d\rangle=\langle\bar{q} q\rangle$.
} 


$$
\Phi\left(x_{\perp}\right)=\frac{|q H|}{2} x^{1} x^{2}
$$

is the so-called Schwinger phase, and the other quantities are

$$
p_{\|}^{2}=p_{0}^{2}-p_{3}^{2}, \quad p_{\perp}^{2}=p_{1}^{2}+p_{2}^{2}, \quad s_{\perp}=\operatorname{sign}(q H) .
$$

The point is that the summation over the Landau levelsassociated with the magnetic field-has already been performed in $\Delta^{ \pm}$. In the thermal propagator, there is then only one sum left: the one induced by finite temperature. This simplifies the calculation considerably. After integration over the momenta, and going from Minkowski to Euclidean space, we obtain

$$
\begin{aligned}
\Delta^{ \pm}(x)= & \frac{|q H|}{(4 \pi)^{\frac{d}{2}}} e^{-s_{\perp}|q H| x_{1} x_{2} / 2} \int_{0}^{\infty} d s \frac{e^{-s M^{2}}}{s \sinh (|q H| s)} \\
& \times \exp \left(-\frac{x_{4}^{2}+x_{3}^{2}}{4 s}-\frac{|q H|\left(x_{1}^{2}+x_{2}^{2}\right)}{4 \tanh (|q H| s)}\right),
\end{aligned}
$$

from which the thermal propagator for the charged pions can be constructed via Eq. (3.7).

Up to one-loop order, the thermal propagator $G(x)$ only has to be evaluated at the origin $x=0$, where it can be decomposed into the $T=0$ contribution and a second piece that refers to finite temperature,

$$
G(0)=\Delta(0)+g_{1}(M, T, H) .
$$

The latter belongs to the class of kinematical Bose functions $g_{r}(M, T, H)$ defined by

$$
\begin{aligned}
g_{r}(M, T, H)= & \frac{T^{d-2 r-2}}{(4 \pi)^{r+1}}|q H| \int_{0}^{\infty} d t \frac{t^{r-\frac{d}{2}}}{\sinh \left(|q H| t / 4 \pi T^{2}\right)} \\
& \times \exp \left(-\frac{M^{2}}{4 \pi T^{2}} t\right)\left[S\left(\frac{1}{t}\right)-1\right]
\end{aligned}
$$

Here, $S(z)$ is the Jacobi theta function,

$$
S(z)=\sum_{n=-\infty}^{\infty} \exp \left(-\pi n^{2} z\right)
$$

The kinematical Bose functions $g_{r}(M, T, H)$ describe the thermodynamic properties of the pions in the presence of magnetic fields. For the effective theory to be consistent, the quantities $T, M$, and $H$ must be small with respect to the underlying QCD scale $\Lambda \approx 1 \mathrm{GeV}$. In this study, we are particularly interested in the chiral limit $M \rightarrow 0$ and the weak magnetic field limit $|q H| \ll T^{2}$.

We proceed with the evaluation of the functions $g_{r}(M, T, H)$. Since the Taylor expansion of the inverse hyperbolic sine starts with

$$
\frac{1}{\sinh (t)}=\frac{1}{t}+\mathcal{O}(t),
$$

we perform the following subtraction in the integrand ${ }^{4}$ :

$$
\begin{aligned}
g_{r}(M, T, H)= & \frac{T^{d-2 r-2}}{(4 \pi)^{r+1}}|q H| \int_{0}^{\infty} d t t^{r-\frac{d}{2}}\left(\frac{1}{\sinh \left(|q H| t / 4 \pi T^{2}\right)}-\frac{4 \pi T^{2}}{|q H| t}\right) \exp \left(-\frac{M^{2}}{4 \pi T^{2}} t\right)\left[S\left(\frac{1}{t}\right)-1\right] \\
& +\frac{T^{d-2 r}}{(4 \pi)^{r}} \int_{0}^{\infty} d t t^{r-\frac{d}{2}-1} \exp \left(-\frac{M^{2}}{4 \pi T^{2}} t\right)\left[S\left(\frac{1}{t}\right)-1\right] .
\end{aligned}
$$

The second term describes pions in zero magnetic field, and it has been evaluated before in Ref. [41]:

$$
\begin{aligned}
g_{r}(M, T, 0)= & 2 \int_{0}^{\infty} \frac{\mathrm{d} \rho}{(4 \pi \rho)^{\frac{d}{2}}} \rho^{r-1} \exp \left(-\rho M^{2}\right) \\
& \times \sum_{n=1}^{\infty} \exp \left(-n^{2} / 4 \rho T^{2}\right) .
\end{aligned}
$$

We thus consider the first term that depends on the magnetic field,

\footnotetext{
${ }^{4}$ Note that we just subtract and re-add a term.
}

$$
\begin{aligned}
\tilde{g}_{r}(M, T, H)= & \frac{|q H|^{\frac{d}{2}-r}}{(4 \pi)^{\frac{d}{2}}} \int_{0}^{\infty} d t t^{r-\frac{d}{2}}\left(\frac{1}{\sinh (t)}-\frac{1}{t}\right) \\
& \times \exp \left(-\frac{M^{2}}{|q H|} t\right)\left[S\left(\frac{|q H|}{4 \pi T^{2} t}\right)-1\right] .
\end{aligned}
$$

Since the analysis of $\tilde{g}_{r}(M, T, H)$ is rather technical, we relegate it to an Appendix. In the same Appendix A, we also discuss the structure of the $T=0$ contribution in the free energy density $z_{0}(M, 0, H)$. Here we just provide the final representation for the quark condensate in the chiral limit and $|q H| \ll T^{2}$. The latter limit is implemented by expanding the various quantities in Eq. (3.6) in the parameter $\epsilon$, 


$$
\epsilon=\frac{|q H|}{T^{2}}
$$

Up to one-loop order, the low-temperature expansion of the quark condensate in the chiral limit and $|q H| \ll T^{2}$ then takes the form

$$
\begin{aligned}
\frac{\langle\bar{q} q\rangle}{\langle 0|\bar{q} q| 0\rangle}= & 1+\frac{|q H| \log 2}{16 \pi^{2} F^{2}}+\left\{\frac{\left|I_{\frac{1}{2}}\right|}{8 \pi^{3 / 2} F^{2}} \sqrt{\epsilon}-\frac{\log 2}{16 \pi^{2} F^{2}} \epsilon\right. \\
& \left.-\frac{a_{1}}{F^{2}} \epsilon^{2}-\frac{a_{2}}{F^{2}} \epsilon^{4}+\mathcal{O}\left(\epsilon^{6}\right)\right\} T^{2} \\
& -\frac{1}{8 F^{2}} T^{2}+\mathcal{O}\left(T^{4}\right)
\end{aligned}
$$

where

$$
\begin{aligned}
& I_{\frac{1}{2}}=\int_{0}^{\infty} d t t^{-1 / 2}\left(\frac{1}{\sinh (t)}-\frac{1}{t}\right) \approx-1.516256 \\
& a_{1}=-\frac{\zeta(3)}{384 \pi^{4}}, \quad a_{2}=\frac{7 \zeta(7)}{98304 \pi^{8}} .
\end{aligned}
$$

The analytical representation for the coefficients $a_{p}$ can be found in Appendix A, along with the numerical values for the first few coefficients $a_{1}, \ldots, a_{5}$ in Table II. The series at finite temperature in nonzero magnetic field is thus dominated by the square-root term $\propto \sqrt{\epsilon}$, followed by a term linear in $\epsilon$. The remaining corrections involve even powers of $\epsilon$.

\section{DISCUSSION AND COMPARISON OF SERIES}

We now discuss and compare our series, Eq. (3.21), with the two results in the literature. The temperatureindependent contribution in the quark condensate that involves the magnetic field,

$$
\frac{|q H| \log 2}{16 \pi^{2} F^{2}}
$$

is the Shushpanov-Smilga term derived a long time ago [33], and later confirmed in Refs. [8-13], among others. However, comparing our leading temperature-dependent contribution,

$$
\frac{\sqrt{|q H|} T}{8 \pi^{3 / 2} F^{2}}\left|I_{\frac{1}{2}}\right|
$$

with the respective leading terms in the two published series, Eq. (2.2),

$$
-\frac{7 \sqrt{|q H|} T}{48 \pi F^{2}}
$$

and Eq. (2.3),

$$
\frac{5 \sqrt{|q H|} T}{48 \pi F^{2}}
$$

we observe disagreement with either result. Still, it is interesting to note that the leading term obtained by Andersen, Eq. (4.4), numerically almost coincides with ours,

$$
\frac{5}{48 \pi} \approx 0.0331573, \quad \frac{1}{8 \pi^{3 / 2}}\left|I_{\frac{1}{2}}\right| \approx 0.0340375 .
$$

In particular, it is also positive. As far as higher-order contributions are concerned, we cannot confirm the emergence of logarithmic terms of the form $H \log \left(H / T^{2}\right)$ as suggested in Refs. [8-11]. Moreover, since the leading term of the Agasian series, Eq. (4.3), comes with the wrong sign, the series clearly fails to describe the quark condensate in the weak magnetic field limit.

To underline the correctness of our series, we perform a simple numerical test. First of all, we establish the connection between our kinematical functions and those in the literature. The representation for the kinematical functions used by the authors of Refs. [8-13] is the same as the one used in Ref. [42], where numerical data are available. The relevant Bose function for the quark condensate in the chiral limit reads

$$
\begin{aligned}
R(0, T, H)= & \frac{\epsilon T^{2}}{2 \pi} \sum_{k=0}^{\infty} \int_{-\infty}^{\infty} \frac{d z}{2 \pi} \frac{1}{\sqrt{z^{2}+(2 k+1) \epsilon}} \\
& \times \frac{1}{\exp \left[\sqrt{z^{2}+(2 k+1) \epsilon}\right]-1} .
\end{aligned}
$$

Using Table I of Ref. [42], we have verified that the connection between the kinematical function $R(0, T, H)$ and our representation $\tilde{g}_{1}(0, T, H)$,

$$
\begin{aligned}
\tilde{g}_{1}(0, T, H)= & \frac{\epsilon T^{2}}{16 \pi^{2}} \int_{0}^{\infty} d t t^{-1}\left(\frac{1}{\sinh (\epsilon t / 4 \pi)}-\frac{4 \pi}{\epsilon t}\right) \\
& \times\left[S\left(\frac{1}{t}\right)-1\right],
\end{aligned}
$$

is given $b y^{5}$

$$
\frac{R(0, T, H)}{T^{2}}-\frac{1}{12}=\frac{\tilde{g}_{1}(0, T, H)}{T^{2}} .
$$

Apart from this numerical consistency check, in Appendix B we analytically derive the above identity.

Having established equivalence between previous analyses and ours through Eq. (4.8), any discrepancies in the weak magnetic field limit $|q H| \ll T^{2}$ can be traced back to

\footnotetext{
${ }^{5}$ Note that the ratio $|q H| / T^{2}=\epsilon$, both in $R(0, T, H)$ and $\tilde{g}_{1}(0, T, H)$, is arbitrary-we are not necessarily referring to the weak magnetic field limit $|q H| \ll T^{2}$.
} 
TABLE I. Leading terms in our series (3.21) for the finite-temperature quark condensate in the limit $|q H| \ll T^{2}$. The $\sqrt{\epsilon}$ term provides a very good approximation for the exact result, and the series converges rapidly. The notation is explained in the text.

\begin{tabular}{lcccc}
\hline \hline$\epsilon$ & $-\tilde{g}_{1} / T^{2}$ & $\mathcal{O}(\sqrt{\epsilon})$ & $\mathcal{O}(\epsilon)$ & $\mathcal{O}\left(\epsilon^{2}\right)$ \\
\hline 0.1 & 0.0103249857050 & 0.01076360492 & 0.01032466434 & 0.0103249857058 \\
0.05 & 0.00739162808212 & 0.007611018032 & 0.007391547742 & 0.00739162808217 \\
0.01 & 0.00335985989497 & 0.003403750739 & 0.003359856681 & 0.00335985989497 \\
0.005 & 0.00238486900367 & 0.002406815229 & 0.002384868200 & 0.00238486900367 \\
0.001 & 0.00107197111872 & 0.001076360492 & 0.001071971087 & 0.00107197111872 \\
0.0005 & 0.000758907108297 & 0.0007611018032 & 0.0007589071003 & 0.000758907108297 \\
0.0001 & 0.000339936133675 & 0.0003403750739 & 0.0003399361334 & 0.000339936133675 \\
\hline \hline
\end{tabular}

the expansion of the kinematical functions in the parameter $\epsilon=|q H| / T^{2}$.

We now perform a numerical test that consists of comparing our series with the exact result. More precisely, we consider successive approximations in the brace ${ }^{6}$

$$
\left\{\frac{\left|I_{\frac{1}{2}}\right|}{8 \pi^{3 / 2} F^{2}} \sqrt{\epsilon}-\frac{\log 2}{16 \pi^{2} F^{2}} \epsilon-\frac{a_{1}}{F^{2}} \epsilon^{2}+\mathcal{O}\left(\epsilon^{4}\right)\right\}
$$

of the expansion (3.21), and we compare them with the exact result given by the Bose function

$$
-\frac{\tilde{g}_{1}(0, T, H)}{T^{2}} .
$$

In Table I, we provide numerical data referring to the terms in the brace [Eq. (4.9)]. The first entry, $\mathcal{O}(\sqrt{\epsilon})$, corresponds to the leading contribution. In the second entry, $\mathcal{O}(\epsilon)$, we also include the term linear in $\epsilon$, while in the third entry, $\mathcal{O}\left(\epsilon^{2}\right)$, we include all three terms displayed in Eq. (4.9). We notice a clear hierarchy: the $\sqrt{\epsilon}$ term yields a very good leading approximation, while subsequent terms are heavily suppressed-our series hence converges very fast.

A final remark concerns the structure of the lowtemperature series and the various limits involved. First, all three series refer to the chiral limit $M \rightarrow 0$. Then, in our low-temperature expansion (3.21), terms are ordered according to ascending powers of $T$, while the accompanying coefficients depend on the ratio $\epsilon=|q H| / T^{2}$. At zero temperature, the series (3.21) reduces to the ShushpanovSmilga term as it should, and the dominant contribution in the low-temperature expansion is of order $T^{2}$.

On the other hand, as one approaches zero temperature, the Agasian series Eq. (2.2) formally reduces to

$$
\frac{\mathcal{C}|q H|}{16 \pi^{2} F^{2}}, \quad \mathcal{C}=\log 2-2 \gamma_{E}+2 \log 4 \pi+\frac{1}{3},
$$

\footnotetext{
${ }^{6}$ Note that each term in the brace has to be multiplied by a factor of $F^{2}$ in order to make the comparison with $-\tilde{g}_{1} / T^{2}$, which is dimensionless.
}

which contradicts the original Shushpanov-Smilga result [33]. According to Refs. [8-11], the extra terms $-2 \gamma_{E}+$ $2 \log 4 \pi+\frac{1}{3}$ originate from the expansion of the quark condensate at finite temperature: they should therefore disappear in the limit $T \rightarrow 0$.

\section{CONCLUSIONS}

The quark condensate at low temperatures, small pion masses, and weak magnetic fields has been studied up to two-loop order in the literature. It was not our intention to contribute to the well-explored phenomena concerning hadrons in magnetic fields that include (inverse) magnetic catalysis. Rather, our point was to draw attention to discrepancies between two published one-loop results, Refs. [8-13], concerning the low-temperature expansion of the quark condensate in the chiral limit in weak magnetic fields.

Our approach relies on chiral perturbation theory, but we have used an alternative representation for the kinematical Bose functions-different from the representations used in Refs. [8-13]. Remarkably, we find that the leading term at finite temperature in the expansion of the quark condensate in a weak magnetic field $\left(|q H| \ll T^{2}\right)$, and in the chiral limit, does not coincide with either of the two published terms. As far as higher-order corrections are concerned, our approach allows for a systematic derivation of these contributions that illuminates the structure of the series.

The low-temperature series is dominated by a squareroot term $\sqrt{|q H| / T^{2}}$ that is positive, much like the (zerotemperature) Shushpanov-Smilga term. The next term is linear in $|q H| / T^{2}$ and negative, while subsequent corrections involve even powers of $|q H| / T^{2}$ with alternating signs. Higher-order terms are heavily suppressed, such that our series converges very rapidly.

Invoking the exact one-loop expression for the quark condensate-valid for arbitrary ratio $|q H| / T^{2}$-we have numerically verified that our expansion correctly describes the quark condensate in weak magnetic fields. We have also observed that the series published in Refs. [8-11] fail to approximate the exact result. 


\section{ACKNOWLEDGMENTS}

The author thanks R. A. Sáenz for helpful comments.

\section{APPENDIX A: EXPLICIT CALCULATIONS}

In this Appendix, we first discuss the free energy density at zero temperature. We then consider the kinematical Bose functions $\tilde{g}_{r}(M, T, H)$ in the chiral limit and analyze their behavior in weak magnetic fields $\left(|q H| \ll T^{2}\right)$. Collecting results, we provide the representation for the quark condensate in the chiral limit and $|q H| \ll T^{2}$. Finally, we show how to extract the leading terms in the expansion of the quark condensate in a straightforward way.

\section{Zero temperature}

The free energy density at zero temperature, up to oneloop order, amounts to

$$
\begin{aligned}
z_{0}(M, 0, H)= & -F^{2} M^{2}-\left(l_{3}+h_{1}\right) M^{4}+4 h_{2}|q H|^{2} \\
& +\frac{1}{2} M^{4} \lambda+I_{1}+I_{2}+\mathcal{O}\left(p^{6}\right), \\
I_{1}= & -\frac{|q H|^{\frac{d}{2}}}{(4 \pi)^{\frac{d}{2}}} \int_{0}^{\infty} d t t^{-\frac{d}{2}-1} \exp \left(-\frac{M^{2}}{|q H|} t\right), \\
I_{2}= & -\frac{|q H|^{\frac{d}{2}}}{(4 \pi)^{\frac{d}{2}}} \int_{0}^{\infty} d t t^{-\frac{d}{2}}\left(\frac{1}{\sinh (t)}-\frac{1}{t}\right) \\
& \times \exp \left(-\frac{M^{2}}{|q H|} t\right) .
\end{aligned}
$$

The integral $I_{1}$ can be written as

$$
I_{1}=M^{4} \lambda-\frac{M^{4}}{64 \pi^{2}},
$$

where $\lambda$

$$
\begin{aligned}
\lambda & =\frac{1}{2}(4 \pi)^{-\frac{d}{2}} \Gamma\left(1-\frac{1}{2} d\right) M^{d-4} \\
& =\frac{M^{d-4}}{16 \pi^{2}}\left[\frac{1}{d-4}-\frac{1}{2}\left\{\ln 4 \pi+\Gamma^{\prime}(1)+1\right\}+\mathcal{O}(d-4)\right]
\end{aligned}
$$

contains a pole at $d=4$. It should be stressed that factors of $|q H|$ cancel: the integral $I_{1}$ does not depend on the magnetic field. The UV divergence in $I_{1}$-along with the UV divergence in the term $\frac{1}{2} M^{4} \lambda$ of Eq. (A1) — can be absorbed into the next-to-leading-order effective constants $l_{3}$ and $h_{1}$ in the standard manner, i.e., in chiral perturbation theory where no magnetic field is present (for details, see, e.g., Ref. [37]).

The integral $I_{2}$, that does depend on the magnetic field, also diverges in the limit $d \rightarrow 4$. The singularity is proportional to $|q H|^{2}$ and can be absorbed into the next-toleading-order effective constant $h_{2}$. Explicitly, we subtract the next Taylor term in the expansion of the inverse hyperbolic sine in $I_{2}$, such that the integral

$-\frac{|q H|^{\frac{d}{2}}}{(4 \pi)^{\frac{d}{2}}} \int_{0}^{\infty} d t t^{-\frac{d}{2}}\left(\frac{1}{\sinh (t)}-\frac{1}{t}+\frac{t}{6}\right) \exp \left(-\frac{M^{2}}{|q H|} t\right)$

becomes finite if one approaches the physical dimension $d \rightarrow 4$. The remainder,

$$
\hat{I}_{2}=\frac{|q H|^{\frac{d}{2}}}{6(4 \pi)^{\frac{d}{2}}} \int_{0}^{\infty} d t t^{-\frac{d}{2}+1} \exp \left(-\frac{M^{2}}{|q H|} t\right)
$$

can be expressed in terms of $\lambda$ as

$$
\hat{I}_{2}=-\frac{|q H|^{2}}{3} \lambda-\frac{|q H|^{2}}{96 \pi^{2}} .
$$

Gathering results, the renormalized free energy density at zero temperature takes the form

$$
\begin{aligned}
z_{0}(M, 0, H) & \\
= & -F^{2} M^{2}+\frac{M^{4}}{64 \pi^{2}}\left(\bar{l}_{3}-4 \bar{h}_{1}-1\right)+\frac{|q H|^{2}}{96 \pi^{2}}\left(\bar{h}_{2}-1\right) \\
& -\frac{|q H|^{2}}{16 \pi^{2}} \int_{0}^{\infty} d t t^{-2}\left(\frac{1}{\sinh (t)}-\frac{1}{t}+\frac{t}{6}\right) \exp \left(-\frac{M^{2}}{|q H|} t\right) \\
& +\mathcal{O}\left(p^{6}\right) .
\end{aligned}
$$

Up to the factors $\gamma_{3} / 32 \pi^{2}, \delta_{1} / 32 \pi^{2}$, and $\delta_{2} / 32 \pi^{2}$, the constants $\bar{l}_{3}, \bar{h}_{1}$, and $\bar{h}_{2}$ are the running coupling constants at the fixed renormalization scale $\mu=M_{\pi}$, where $M_{\pi} \approx$ $139.6 \mathrm{MeV}$ is the physical pion mass (for details, see, e.g., Ref. [41]).

\section{Finite temperature}

We now turn to finite temperature, where the kinematical Bose functions

$$
\begin{aligned}
\tilde{g}_{r}(M, T, H)= & \frac{|q H|^{\frac{d}{2}-r}}{(4 \pi)^{\frac{d}{2}}} \int_{0}^{\infty} d t t^{r-\frac{d}{2}}\left(\frac{1}{\sinh (t)}-\frac{1}{t}\right) \\
& \times \exp \left(-\frac{M^{2}}{|q H|} t\right)\left[S\left(\frac{|q H|}{4 \pi T^{2} t}\right)-1\right]
\end{aligned}
$$

become relevant. Our analysis proceeds along the lines of Ref. [43]. From the very start, we refer to the chiral limit that we implement by $M \rightarrow 0$, while keeping $T$ and $|q H|$ fixed. Changing integration variables, and defining $\epsilon=|q H| / T^{2}$, we first write 


$$
\begin{aligned}
\tilde{g}_{r}(0, T, H) \\
=\frac{\epsilon}{(4 \pi)^{r+1}} T^{d-2 r} \int_{0}^{\infty} d t t^{-\frac{d}{2}+r}\left(\frac{1}{\sinh (\epsilon t / 4 \pi)}-\frac{4 \pi}{\epsilon t}\right) \\
\quad \times\left[S\left(\frac{1}{t}\right)-1\right] .
\end{aligned}
$$

The integral is split into two pieces, namely $0 \leq t \leq 1$ and $1 \leq t<\infty$. In the second interval, we use the Jacobi identity

$$
S(t)=\frac{1}{\sqrt{t}} S(1 / t)
$$

and change the integration variable $t \rightarrow 1 / t$. This then leads to

$$
\begin{aligned}
\tilde{g}_{r}(0, T, H)= & \frac{\epsilon}{(4 \pi)^{r+1}} T^{d-2 r} \int_{0}^{1} d t t^{-\frac{d}{2}+r}\left(\frac{1}{\sinh (\epsilon t / 4 \pi)}-\frac{4 \pi}{\epsilon t}\right) \\
& \times\left[S\left(\frac{1}{t}\right)-1\right]+\frac{\epsilon}{(4 \pi)^{r+1}} T^{d-2 r}\left\{I_{A}+I_{B}+I_{C}\right\},
\end{aligned}
$$

with

$$
\begin{aligned}
& I_{A}=\int_{0}^{1} d t t^{\frac{d}{2}-r-\frac{5}{2}}\left(\frac{1}{\sinh (\epsilon / 4 \pi t)}-\frac{4 \pi t}{\epsilon}\right)\left[S\left(\frac{1}{t}\right)-1\right] \\
& I_{B}=\int_{0}^{1} d t t^{\frac{d}{2}-r-\frac{5}{2}}\left(\frac{1}{\sinh (\epsilon / 4 \pi t)}-\frac{4 \pi t}{\epsilon}\right) \\
& I_{C}=-\int_{0}^{1} d t t^{\frac{d}{2}-r-2}\left(\frac{1}{\sinh (\epsilon / 4 \pi t)}-\frac{4 \pi t}{\epsilon}\right)
\end{aligned}
$$

The integral $I_{B}$ we decompose as

$$
\begin{aligned}
I_{B}= & \int_{0}^{\infty} d t t^{\frac{d}{2}-r-\frac{5}{2}}\left(\frac{1}{\sinh (\epsilon / 4 \pi t)}-\frac{4 \pi t}{\epsilon}\right) \\
& -\int_{1}^{\infty} d t t^{\frac{d}{2}-r-\frac{5}{2}}\left(\frac{1}{\sinh (\epsilon / 4 \pi t)}-\frac{4 \pi t}{\epsilon}\right) .
\end{aligned}
$$

After a few trivial manipulations, we end up with

$$
\begin{aligned}
I_{B} & =I_{B 1}+I_{B 2}, \\
I_{B 1} & =\frac{\epsilon^{\frac{d}{2}-r-\frac{3}{2}}}{(4 \pi)^{\frac{d}{2}-r-\frac{3}{2}}} \int_{0}^{\infty} d t t^{-\frac{d}{2}+r+\frac{1}{2}}\left(\frac{1}{\sinh (t)}-\frac{1}{t}\right), \\
I_{B 2} & =-\int_{0}^{1} d t t^{-\frac{d}{2}+r+\frac{1}{2}}\left(\frac{1}{\sinh (\epsilon t / 4 \pi)}-\frac{4 \pi}{\epsilon t}\right) .
\end{aligned}
$$

The integral $I_{C}$ is processed in an analogous way, with the result

$$
\begin{aligned}
I_{C} & =I_{C 1}+I_{C 2}, \\
I_{C 1} & =-\frac{\epsilon^{\frac{d}{2}-r-1}}{(4 \pi)^{\frac{d}{2}-r-1}} \int_{0}^{\infty} d t t^{-\frac{d}{2}+r}\left(\frac{1}{\sinh (t)}-\frac{1}{t}\right), \\
I_{C 2} & =\int_{0}^{1} d t t^{-\frac{d}{2}+r}\left(\frac{1}{\sinh (\epsilon t / 4 \pi)}-\frac{4 \pi}{\epsilon t}\right) .
\end{aligned}
$$

\section{Representation for the quark condensate}

We now focus on the quark condensate in the chiral limit,

$\langle\bar{q} q\rangle=\langle 0|\bar{q} q| 0\rangle\left[1-\frac{1}{F^{2}} \frac{\partial}{\partial M^{2}}\left(z-z_{0}(M, 0,0)\right)\right]_{M^{2}=0}$,

where

$$
z=z_{0}(M, 0, H)-\frac{3}{2} g_{0}(M, T, 0)-\tilde{g}_{0}(M, T, H)+\mathcal{O}\left(p^{6}\right)
$$

is the (total) free energy density and

$$
z_{0}(M, 0,0)=-F^{2} M^{2}+\frac{M^{4}}{64 \pi^{2}}\left(\bar{l}_{3}-4 \bar{h}_{1}-1\right)
$$

is the $T=0$ contribution that is independent of the magnetic field. Accordingly, the one-loop representation for the quark condensate in the chiral limit reads

$$
\begin{aligned}
\frac{\langle\bar{q} q\rangle}{\langle 0|\bar{q} q| 0\rangle}= & 1-\frac{|q H|}{16 \pi^{2} F^{2}} \int_{0}^{\infty} d t t^{-1}\left(\frac{1}{\sinh (t)}-\frac{1}{t}\right) \\
& -\frac{3 g_{1}(0, T, 0)}{2 F^{2}}-\frac{\tilde{g}_{1}(0, T, H)}{F^{2}}+\mathcal{O}\left(p^{4}\right) .
\end{aligned}
$$

The first line refers to zero temperature, and the second line refers to finite temperature.

Our final task is to explore the weak magnetic field limit $|q H| \ll T^{2}$ that we obtain by expanding in the parameter $\epsilon$

$$
\epsilon=\frac{|q H|}{T^{2}}
$$

A common factor in the various integrands considered in Sec. A 2 of this Appendix is

$$
\frac{1}{\sinh (\epsilon t / 4 \pi)}-\frac{4 \pi}{\epsilon t}
$$

which we expand into

$\frac{1}{\sinh (\epsilon t / 4 \pi)}-\frac{4 \pi}{\epsilon t}=c_{1} t \epsilon+c_{2} t^{3} \epsilon^{3}+c_{3} t^{5} \epsilon^{5}+\mathcal{O}\left(\epsilon^{7}\right)$. 
The first few Taylor coefficients read

$$
\begin{aligned}
& c_{1}=-\frac{1}{24 \pi} \approx-1.33 \times 10^{-2}, \\
& c_{2}=\frac{7}{23040 \pi^{3}} \approx 9.80 \times 10^{-6}, \\
& c_{3}=-\frac{31}{15482880 \pi^{5}} \approx-6.54 \times 10^{-9}, \\
& c_{4}=\frac{127}{9909043200 \pi^{7}} \approx 4.24 \times 10^{-12}, \\
& c_{5}=-\frac{73}{896909967360 \pi^{9}} \approx-2.73 \times 10^{-15} .
\end{aligned}
$$

Introducing the quantities $\tilde{\alpha}_{p}, \hat{\alpha}_{p}$, and $\beta_{p}$ by

$$
\begin{aligned}
& \tilde{\alpha}_{p}=\int_{0}^{1} d t c_{p} t^{2 p-2}\left[S\left(\frac{1}{t}\right)-1\right], \\
& \hat{\alpha}_{p}=\int_{0}^{1} d t c_{p} t^{-2 p-\frac{1}{2}}\left[S\left(\frac{1}{t}\right)-1\right], \\
& \beta_{p}=\int_{0}^{1} d t c_{p}\left(t^{-1}-t^{-\frac{1}{2}}\right) t^{2 p-1},
\end{aligned}
$$

and defining the coefficients $a_{p}$ as

$$
a_{p}=\frac{\tilde{\alpha}_{p}+\hat{\alpha}_{p}+\beta_{p}}{16 \pi^{2}},
$$

the low-temperature representation for the quark condensate in the chiral limit and $|q H| \ll T^{2}$ then takes the form

$$
\begin{aligned}
\frac{\langle\bar{q} q\rangle}{\langle 0|\bar{q} q| 0\rangle}= & 1+\frac{|q H| \log 2}{16 \pi^{2} F^{2}}+\left\{\frac{\left|I_{\frac{1}{2}}\right|}{8 \pi^{3 / 2} F^{2}} \sqrt{\epsilon}-\frac{\log 2}{16 \pi^{2} F^{2}} \epsilon\right. \\
& \left.-\frac{a_{1}}{F^{2}} \epsilon^{2}-\frac{a_{2}}{F^{2}} \epsilon^{4}+\mathcal{O}\left(\epsilon^{6}\right)\right\} T^{2} \\
& -\frac{1}{8 F^{2}} T^{2}+\mathcal{O}\left(T^{4}\right) .
\end{aligned}
$$

The integral $I_{\frac{1}{2}}$ amounts to

$$
I_{\frac{1}{2}}=\int_{0}^{\infty} d t t^{-1 / 2}\left(\frac{1}{\sinh (t)}-\frac{1}{t}\right) \approx-1.516256,
$$

while numerical values for the first few coefficients $a_{p}$ in the above expansion are provided in Table II.

Processing integrals in the same manner as described in the previous section, and using the identity

$$
\frac{2}{\pi^{\frac{z}{2}}} \Gamma\left(\frac{z}{2}\right) \zeta(z)=\int_{0}^{\infty} d t t^{\frac{z}{2}-1}[S(t)-1]
$$

we can express the coefficients $a_{p}$ in terms of the Riemann $\zeta$ function as
TABLE II. Numerical values for the coefficients $a_{p}$ defined by Eq. (A25).

\begin{tabular}{lc}
\hline \hline$p$ & $a_{p}$ \\
\hline 1 & $-3.21361844712 \times 10^{-5}$ \\
2 & $7.56726355863 \times 10^{-9}$ \\
3 & $-8.00051395855 \times 10^{-12}$ \\
4 & $1.87869037118 \times 10^{-14}$ \\
5 & $-7.80774216239 \times 10^{-17}$ \\
\hline \hline
\end{tabular}

$$
a_{p}=\frac{c_{p}}{8 \pi^{2 p+\frac{3}{2}}} \Gamma\left(2 p-\frac{1}{2}\right) \zeta(4 p-1) .
$$

The final representation for the quark condensate in the chiral limit and $|q H| \ll T^{2}$ thus reads

$$
\begin{aligned}
\frac{\langle\bar{q} q\rangle}{\langle 0|\bar{q} q| 0\rangle}= & 1-\frac{1}{8 F^{2}} T^{2}+\frac{|q H| \log 2}{16 \pi^{2} F^{2}} \\
& +\left\{\frac{\left|I_{\frac{1}{2}}\right|}{8 \pi^{3 / 2} F^{2}} \sqrt{\epsilon}-\frac{\log 2}{16 \pi^{2} F^{2}} \epsilon+\frac{\zeta(3)}{384 \pi^{4} F^{2}} \epsilon^{2}\right. \\
& \left.-\frac{7 \zeta(7)}{98304 \pi^{8} F^{2}} \epsilon^{4}+\mathcal{O}\left(\epsilon^{6}\right)\right\} T^{2}+\mathcal{O}\left(T^{4}\right) .
\end{aligned}
$$

\section{Straightforward derivation of the leading terms}

In order to readily derive the leading terms in the quark condensate in the chiral limit and $|q H| \ll T^{2}$, we consider the relevant Bose function

$$
\begin{aligned}
\tilde{g}_{1}(0, T, H)= & \frac{|q H|}{16 \pi^{2}} \int_{0}^{\infty} d t t^{-1}\left(\frac{1}{\sinh (t)}-\frac{1}{t}\right) \\
& \times\left[S\left(\frac{|q H|}{4 \pi T^{2} t}\right)-1\right],
\end{aligned}
$$

that we write as

$$
\begin{aligned}
\tilde{g}_{1}(0, T, H)= & -\frac{|q H|}{16 \pi^{2}} \int_{0}^{\infty} d t t^{-1}\left(\frac{1}{\sinh (t)}-\frac{1}{t}\right) \\
& +\frac{\sqrt{|q H| T}}{8 \pi^{3 / 2}} \int_{0}^{\infty} d t t^{-1 / 2}\left(\frac{1}{\sinh (t)}-\frac{1}{t}\right) \\
& \times S\left(\frac{4 \pi T^{2}}{|q H|} t\right) .
\end{aligned}
$$

Note that the Jacobi theta function

$$
S(z)=\sum_{n=-\infty}^{\infty} \exp \left(-\pi n^{2} z\right)
$$

satisfies the identity 


$$
S(z)=\frac{1}{\sqrt{z}} S\left(\frac{1}{z}\right)
$$

The integral in the first line, Eq. (A32), is known analytically,

$$
\int_{0}^{\infty} d t t^{-1}\left(\frac{1}{\sinh (t)}-\frac{1}{t}\right)=-\log 2,
$$

and gives rise to the correction linear in $\epsilon$ in Eq. (A26). Regarding the second line, Eq. (A32), in the limit $|q H| \ll T^{2}$, all contributions in the Jacobi theta function-except $n=0$ - are exponentially suppressed: the corresponding integral hence reduces to $I_{\frac{1}{2}}$, Eq. (A27), and we immediately obtain the leading temperature-dependent term in the weak magnetic field expansion $|q H| \ll T^{2}$ in the chiral limit,

$$
\frac{\sqrt{|q H|} T}{8 \pi^{3 / 2} F^{2}}\left|I_{\frac{1}{2}}\right| .
$$

\section{APPENDIX B: REPRESENTATIONS FOR BOSE-EINSTEIN FUNCTIONS}

In this Appendix, we derive the analytical connection between two alternative representations for the kinematical functions that refer to the Bose gas. In either case, the basic object of interest is the thermal propagator for the charged pions in the background of a magnetic field. We first consider the approach taken by the authors of Refs. [8-13]. In Appendix A of Ref. [13], the starting point is the dimensionally regularized sum-integral

$$
\begin{aligned}
& \mathcal{R}(M, T, H) \\
& =\frac{|q H|}{2 \pi} \sum_{m=0}^{\infty} T \sum_{P_{0}=2 \pi n T}\left(\frac{e^{\gamma_{E}} \Lambda^{2}}{4 \pi}\right)^{\varepsilon} \\
& \quad \times \int \frac{d^{d_{s}-2} p}{(2 \pi)^{d_{s}-2}} \frac{1}{P_{0}^{2}+p_{z}^{2}+M^{2}+(2 m+1)|q H|}
\end{aligned}
$$

that describes the thermal propagator for the charged pions. The sum over $m$ is due to the Landau levels induced by the magnetic field, while the sum over $n$ is over the Matsubara frequencies related to finite temperature. The various symbols occurring in Eq. (B1) are defined in Appendix A of Ref. [13], and the solution of Eq. (B1) is given by Eq. (A8) in that reference. Here we only need the nonzero-temperature part $R(M, T, H)$ of the (full) thermal propagator $\mathcal{R}(M, T, H)$, which, in the chiral limit, amounts to

$$
\begin{aligned}
R(0, T, H)= & \frac{\epsilon T^{2}}{2 \pi} \sum_{k=0}^{\infty} \int_{-\infty}^{\infty} \frac{d z}{2 \pi} \frac{1}{\sqrt{z^{2}+(2 k+1) \epsilon}} \\
& \times \frac{1}{\exp \left[\sqrt{z^{2}+(2 k+1) \epsilon}\right]-1} .
\end{aligned}
$$

Note that the physical limit $d_{s} \rightarrow 3(\varepsilon \rightarrow 0)$ has been taken. $^{7}$

The approach pursued in the present article, on the other hand, is based on the coordinate representation of the thermal propagator, where the periodicity condition induced by finite temperature corresponds to the sum over $n$ in the Euclidean time direction,

$$
G(x)=\sum_{n=-\infty}^{\infty} \Delta\left(\vec{x}, x_{4}+n \beta\right), \quad \beta=\frac{1}{T} .
$$

According to Sec. III, the explicit expression for the zero-temperature propagator of the charged pions $\Delta^{ \pm}(x)$ is given by

$$
\begin{aligned}
\Delta^{ \pm}(x)= & \frac{|q H|}{(4 \pi)^{\frac{d}{2}}} e^{-s_{\perp}|q H| x_{1} x_{2} / 2} \int_{0}^{\infty} d s \frac{e^{-s M^{2}}}{s \sinh (|q H| s)} \\
& \times \exp \left(-\frac{x_{4}^{2}+x_{3}^{2}}{4 s}-\frac{|q H|\left(x_{1}^{2}+x_{2}^{2}\right)}{4 \tanh (|q H| s)}\right) .
\end{aligned}
$$

It should be noted that we have used the representation (D.15) for the zero-temperature propagator provided in Ref. [32], where the sum over $m$ related to the Landau levels has already been performed. As described in Sec. III of the present article, the relevant quantity for the quark condensate at one-loop order is the thermal propagator $G(x)$ evaluated at the origin,

$$
G(0)=\Delta(0)+g_{1}(M, T, H) .
$$

In the above decomposition, $\Delta(0)$ is the zero-temperature propagator, while the finite-temperature part is given by the Bose-Einstein function (or, equivalently, kinematical function) $g_{1}(M, T, H)$,

$$
\begin{aligned}
g_{1}(M, T, H)= & \frac{|q H|}{(4 \pi)^{2}} \int_{0}^{\infty} d t \frac{t^{-1}}{\sinh \left(|q H| t / 4 \pi T^{2}\right)} \\
& \times \exp \left(-\frac{M^{2}}{4 \pi T^{2}} t\right)\left[S\left(\frac{1}{t}\right)-1\right],
\end{aligned}
$$

with the Jacobi theta function

$$
S(z)=\sum_{n=-\infty}^{\infty} \exp \left(-\pi n^{2} z\right) .
$$

In the chiral limit, the correspondence between the two representations $R(0, T, H)$ and $g_{1}(0, T, H)$ is thus given by the identity

\footnotetext{
${ }^{7}$ The quantity $\varepsilon=\left(3-d_{s}\right) / 2$ (where $d_{s}$ is the spatial dimension) should not be confused with our expansion parameter $\epsilon=|q H| / T^{2}$.
} 


$$
R(0, T, H)=g_{1}(0, T, H)
$$

based on the fact that we have calculated the same quantity-namely, the thermal propagator-using two different methods.

There is one last detail, though. The kinematical function we use in our comparison of the various series in Sec. IV is $\tilde{g}_{1}(0, T, H)$, and not $g_{1}(0, T, H)$. According to our decomposition, Eq. (3.17), the former describes the purely nonzero magnetic field part, while the latter also involves terms that exclusively depend on temperature (and are independent of the magnetic field). This is why in the representation $R(0, T, H)$, one also has to subtract the purely $T$-dependent (and $H$-independent) part to make the comparison. In the chiral limit, this term is (see Ref. [41] or Appendix A of Ref. [44])

$$
R(0, T, 0)=g_{1}(0, T, 0)=\frac{T^{2}}{12} .
$$

This then leads to the analytical connection between the two representations of Bose-Einstein functions,

$$
\frac{R(0, T, H)}{T^{2}}-\frac{1}{12}=\frac{\tilde{g}_{1}(0, T, H)}{T^{2}} .
$$

[1] G. S. Bali, F. Bruckmann, G. Endrödi, Z. Fodor, S. D. Katz, S. Krieg, A. Schäfer, and K. K. Szabó, J. High Energy Phys. 02 (2012) 044.

[2] V. V. Braguta, P. V. Buividovich, T. Kalaydzhyan, S. V. Kuznetsov, and M. I. Polikarpov, Phys. At. Nucl. 75, 488 (2012).

[3] M. D'Elia, S. Mukherjee, and F. Sanfilippo, Phys. Rev. D 82, 051501 (2010).

[4] M. D'Elia and F. Negro, Phys. Rev. D 83, 114028 (2011).

[5] G. S. Bali, F. Bruckmann, G. Endrödi, Z. Fodor, S. D. Katz, and A. Schäfer, Phys. Rev. D 86, 071502 (2012).

[6] G. S. Bali, F. Bruckmann, M. Constantinou, M. Costa, G. Endrödi, Z. Fodor, S. D. Katz, H. Panagopoulos, and A. Schäfer, Phys. Rev. D 86, 094512 (2012).

[7] V. G. Bornyakov, P. V. Buividovich, N. Cundy, O. A. Kochetkov, and A. Schäfer, Phys. Rev. D 90, 034501 (2014).

[8] N. O. Agasian, Phys. Lett. B 488, 39 (2000).

[9] N. O. Agasian, Phys. At. Nucl. 64, 554 (2001).

[10] N. O. Agasian and I. A. Shushpanov, J. High Energy Phys. 10 (2001) 006.

[11] N. O. Agasian, Phys. At. Nucl. 71, 1967 (2008).

[12] J. O. Andersen, Phys. Rev. D 86, 025020 (2012).

[13] J. O. Andersen, J. High Energy Phys. 10 (2012) 005.

[14] R. Gatto and M. Ruggieri, Phys. Rev. D 83, 034016 (2011).

[15] A. Amador and J. O. Andersen, Phys. Rev. D 88, 025016 (2013).

[16] M. Ferreira, P. Costa, D. P. Menezes, C. Providencia, and N. N. Scoccola, Phys. Rev. D 89, 016002 (2014).

[17] R. Zhang, W. Fu, and Y. Liu, Eur. Phys. J. C 76, 307 (2016).

[18] S. Nam and C.-W. Kao, Phys. Rev. D 83, 096009 (2011).

[19] M. Frasca and M. Ruggieri, Phys. Rev. D 83, 094024 (2011).

[20] V. D. Orlovsky and Y. A. Simonov, J. High Energy Phys. 09 (2013) 136.

[21] V. D. Orlovsky and Y. A. Simonov, Phys. Rev. D 89, 074034 (2014).

[22] G. Endrödi, J. High Energy Phys. 04 (2013) 023.

[23] F. Bruckmann, G. Endrödi, and T. G. Kovács, J. High Energy Phys. 04 (2013) 112.
[24] E.-M. Ilgenfritz, M. Müller-Preussker, B. Petersson, and A. Schreiber, Phys. Rev. D 89, 054512 (2014).

[25] E. J. Ferrer, V. de la Incera, I. Portillo, and M. Quiroz, Phys. Rev. D 89, 085034 (2014).

[26] M. Ferreira, P. Costa, O. Lourenco, T. Frederico, and C. Providencia, Phys. Rev. D 89, 116011 (2014).

[27] A. Haber, F. Preis, and A. Schmitt, Phys. Rev. D 90, 125036 (2014).

[28] J. O. Andersen, W. R. Naylor, and A. Tranberg, J. High Energy Phys. 04 (2014) 187.

[29] N. Mueller and J. M. Pawlowski, Phys. Rev. D 91, 116010 (2015).

[30] G. Endrödi, J. High Energy Phys. 07 (2015) 173.

[31] A. N. Tawfik, A. M. Diab, N. Ezzelarab, and A. G. Shalaby, Adv. High Energy Phys. 2016, 1381479 (2016).

[32] J. O. Andersen, W. R. Naylor, and A. Tranberg, Rev. Mod. Phys. 88, 025001 (2016).

[33] I. A. Shushpanov and A. V. Smilga, Phys. Lett. B 402, 351 (1997).

[34] J. Gasser and H. Leutwyler, Phys. Lett. B 184, 83 (1987).

[35] H. Leutwyler, in Hadron Physics 94: Topics on the Structure and Interaction of Hadronic Systems, edited by V.E. Herscovitz, C. A. Z. Vasconcellos, and E. Ferreira (World Scientific, Singapore, 1995), p. 1.

[36] S. Scherer, Adv. Nucl. Phys. 27, 277 (2003).

[37] J. Gasser and H. Leutwyler, Ann. Phys. (N.Y.) 158, 142 (1984).

[38] C. P. Hofmann, J. Stat. Mech. (2016) 093102.

[39] C. P. Hofmann, Nucl. Phys. B916, 254 (2017).

[40] I. A. Shovkovy, Lect. Notes Phys. 871, 13 (2013).

[41] P. Gerber and H. Leutwyler, Nucl. Phys. B321, 387 (1989).

[42] D. C. Duarte, R. L. S. Farias, and R. O. Ramos, Phys. Rev. D 84, 083525 (2011).

[43] P. Hasenfratz and H. Leutwyler, Nucl. Phys. B343, 241 (1990).

[44] J. O. Andersen, E. Braaten, and M. Strickland, Phys. Rev. D 63, 105008 (2001). 